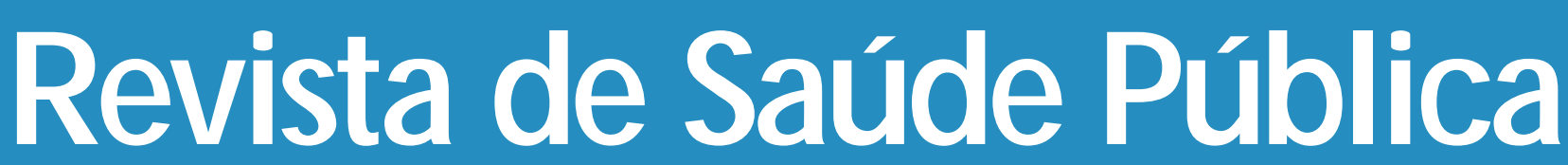

$\begin{array}{llllllll}\mathbf{J} & \mathbf{O} & \mathbf{U} & \mathbf{R} & \mathbf{N} & \mathbf{A} & \mathbf{L}\end{array}$

$0 F$

$\mathbf{P} \mathbf{U}$

B L I C

H E A L T

\title{
Costo-efectividad de intervenciones para insuficiencia renal crónica terminal
}

\section{Cost-effectiveness of interventions for end-stage renal disease}

\section{Armando Arredondo*, Ricardo Rangel y Esteban de Icaza}

Departamento de Investigación en Costos y Financiamiento para la Salud del Centro de Investigaciones en Sistemas de Salud del Instituto Nacional de Salud Pública. Cuernavaca Morelos, México (A.A.), Departamento de Administración de la Salud de la Faculdad de Medicina de la Universidad de Montreal. Montreal, Canada (R.R., E.I.)

ARRED O NDO Armando, Ricardo Rangel y Esteban de Icaza Costo-efectividad de intervenciones para insuficiencia renal crónica terminal Rev. Saúde Pública, 32 (6): 556-65, 1998

(c) Copyright Faculdade de Saúde Pública da USP. Proibida a reprodução mesmo que parcial sem a devida autorização do Editor Científico. Proibida a utilização de matérias para fins comerciais. All rights reserved. 


\title{
Costo-efectividad de intervenciones para insuficiencia renal crónica terminal
}

\section{Cost-effectiveness of interventions for end-stage renal disease}

\author{
Armando Arredondo*, Ricardo Rangel y Esteban de Icaza \\ Departamento de Investigación en Costos y Financiamiento para la Salud del Centro \\ de Investigaciones en Sistemas de Salud del Instituto Nacional de Salud Pública. \\ Cuernavaca Morelos, México (A.A.), Departamento de Administración de la Salud \\ de la Faculdad de Medicina de la Universidad de Montreal. Montreal, Canada (R.R., E.I.)
}

\begin{abstract}
Objetivo Analisó el costo-efectividad en intervenciones para pacientes con insuficiencia renal crónica terminal (IRCT) en términos de los costos económicos de cada intervención, los años de vida ganados y la calidad de vida que generan tres alternativas comparables y mutuamente excluyentes: diálisis peritoneal contínua ambulatoria (DPCA), la hemodiálisis (HD) y el trasplante renal (TR).

Método El diseño del estudio fue de tipo longitudinal. Los costos de cada intervención se determinaron mediante la técnica de manejo de caso promedio. Las medidas para evaluar los criterios de efectividad elegidos fueron la probabilidad de sobrevida y el Año de Vida Ajustado por Calidad (QALY, Quality Adjusted Life Year) medido por el Indice de Rosser.
\end{abstract}

Resultados

Los costos de manejo anual de caso fueron: diálisis peritoneal \$5,643.07, hemodiálisis $\$ 9,631.60$ y trasplante $\$ 3,021.63$. En cuanto a la efectividad, la sobrevida del injerto de trasplante renal resultó de $89,9 \%$ y $79,6 \%$ a uno y tres años respectivamente, mientras que los pacientes sometidos a DPCA tienen una sobrevida de $86,2 \%$ y $66,9 \%$ a un año y a tres años respectivamente. En cuanto a los QALY's, los resultados para cada intervención fueron: DPCA 0,879 ; HD 0,864; y para el TR 0,978.

Conclusión La intervención más costo-efectiva resultó el trasplante renal con un coeficiente de 3,088.69, seguido de la DPCA y la hemodiálisis, cuyos coeficientes fueron de $6,416.95$ y $11,147.68$ respectivamente. Por lo tanto se recomienda promover y utilizar el trasplante renal como la intervención más costo-efectiva para pacientes con IRCT.

Insuficiencia renal crónica. Análisis de costo-beneficio. Calidad de vida.

\section{Abstract}

Objective The study reports the cost-effectiveness results of end-stage renal disease (ESDR) patients in Mexico in terms of years of life gained and quality of life and the economic cost with regards to three treatment alternatives that could be considered mutually exclusive: continuous peritoneal ambulatory dialysis $(C A P D)$, hemodialysis $(H D)$ and renal transplant $(R T)$.

*Profesor visitante en el Departamento de Administración de la Salud de la Universidad de Montreal. Correspondencia para/Correspondence to: Armando Arredondo-Av. Universidad No 655. Cuernavaca, Mor. México. E-mail: arredona@magellan.umontreal.ca. Recibido en 6/6/1997. Reapresentado en 10/12/1997. Aprobado en 30/4/1998. 
Method The economic costs were calculated by using the average cost case-management methodology and further, the probable of life expectancy and the quality of life were cross-sectionally assessed by means of the Quality Adjusted Life Years (QALY) measured by the Rosser Index.

Results The results show that economic costs in US\$ of the three treatment alternatives were: $C A P D \$ 5,643.07, H D \$ 9,631.60$, and $R T \$ 3,021.63$. The probability of life expectancy for CAPD and RT for the first and third year were: $86.2 \%$ and $66.9 \%$, and $89.9 \%$ and $79.6 \%$, respectively. The QALY scores for patients were: CAPD 0.8794, HD 0.8640, and RT 0.9783.

Conclusion The intervention with the highest cost-effectiveness coefficient was the renal transplant (3,088.69), followed by the CAPD (6,416.95) and HD $(11,147.68)$. A significant difference was found between the transplanted patients and patients undergoing dialysis. Finally, this study concluded that the RT offers the least expensive alternative and the greatest number of years of life gained as well as providing significant changes in the quality of life of ESRD patients.

Kidney failure, chronic, economy. Cost-benefit analysis. Quality of life.

\section{Resumo}

Objetivo Analisar o custo-efetividade de intervenções realizadas em pacientes com insuficiência renal crônica terminal (IRCT) quanto a custos econômicos de cada intervenção, anos de vida ganhos e qualidade de vida que geram três alternativas comparáveis e mutuamente excluentes: diálise peritoneal contínua ambulatória (DPCA), hemodiálise (HD) e transplante renal (TR).

Método O desenho do estudo foi do tipo longitudinal. Os custos de cada intervenção foram determinados mediante a técnica de manejo de caso promédio. As medidas para avaliar os critérios de efetividade elegidos foram a probabilidade de sobrevida e o Ano de Vida Ajustado por Qualidade (QALY, Quality Adjusted Life Year) medido pelo Índice de Rosser.

Resultados Os custos do manejo anual de caso foram: diálise peritoneal US\$5,643.07, hemodiálise US\$9,631.60 e transplante US\$3,021.63. Quanto à efetividade, a sobrevida do transplante renal resultou em $89,9 \%$ e $79,6 \%$ para um e três anos, respectivamente, enquanto que os pacientes submetidos a DPCA têm uma sobrevida de $86,2 \%$ e $66,9 \%$ para um ano e três anos, respectivamente. Quanto aos QALY's, os resultados para cada intervenção foram: DPCA 0,879; HD 0,864 ; e para o TR 0,978 .

Conclusão

A intervenção mais custo-efetivo mostrou ser o transplante renal com um coeficiente de 3,088.69 seguido da DPCA e da hemodiálise, cujos coeficientes foram de 6,416.95 e 11,147.68, respectivamente. Recomenda-se promover e utilizar o transplante renal como a intervenção mais custo-efetivo para pacientes com IRCT.

Insuficiência renal crônica, economia.Análise custo-benefício. Qualidade de vida.

\section{INTRO DUCCIÓN}

Los retos de la salud pública actual en el marco de reformas del sector, se encuentran influídos por dos aspectos que no pueden dejarse de lado en la evaluación sobre la producción de los servicios de salud: las necesidades infinitas de la población y la escasez de recursos financieros. Alrededor de esos dos aspectos, surge la necesidad de desarrollar marcos teóricos-metodológicos de referencia, planes de 
acción estratégica, evaluación tanto de la teoría como de la práctica; pero sobre todo surge la necesidad de generar insumos de información que permitan tomar decisiones con la mayor racionalidad para implementar las intervenciones que permitan disminuir, en la medida de lo posible los efectos de la falta de equidad, eficiencia y calidad en la prestación de servicios médicos ${ }^{11}$.

Por otra parte, la generación de información para la toma de decisiones en el uso eficiente de los recursos que se asignan al sector, se deberían plantear en un ámbito de transición tanto de las condiciones epidemiológicas, caracterizadas por cambios importantes en cuanto a demandas de enfermedades crónico-degenerativas vs. infecto-contagiosas; así como de la transición en los sistemas de salud hacia la reorganización en la producción y financiamiento de los servicios de salud a ofrecer ${ }^{15}$. En este contexto, el análisis de costo-efectividad se toma como un trazador de la problemática planteada alrededor de la eficiencia de los sistemas de salud, tomando como intervención de estudio los programas de atención dirigidos a pacientes con insuficiencia renal crónica terminal (IRCT).

En cuanto a la IRCT, se puede decir que no difiere de otras enfermedades crónico degenerativas que han visto su incidencia aumentar significativamente a lo largo de la última década debido a la transición epidemiológica. Es decir, como se mencionó anteriormente, México se encuentra en un proceso de transición epidemiológica en el que las enfermedades no transmisibles adquieren un mayor peso relativo al tiempo que las enfermedades transmisibles continúan causando estragos entre la población, especialmente entre los grupos más pobres y marginados ${ }^{16}$

La incidencia de la IRCT en México se ha venido aproximando, de forma abrupta y descontrolada a niveles semejantes a los encontrados en países industrializados. Actualmente se calculan 40,000 urémicos en México que demandan servicios de atención al sistema de salud en cualquiera de sus modalidades público-privadas ${ }^{14}$. Además, cabe resaltar la importante prevalencia de pacientes diabéticos en México que son responsables por el $25 \%$ de los pacientes con IRCT ${ }^{22}$.

En México los procedimientos que operan médicamente para el tratamiento de la IRCT son la dialisis peritoneal contínua ambulatorial (DPCA), la diálisis en centro hospitalario (diálisis peritoneal intermitente y hemodiálisis) y el transplate renal (TR). Tan sólo en el Instituto Mexicano del Seguro Social hay 13,000 pacientes en DPCA y 1,300 en hemo- diálisis, al tiempo que aparecen 120 nuevos casos por millón de habitantes cada año ${ }^{7}$.

De esta manera, la importancia de la evaluación de los costos y la efectividad de intervenciones, radica en su potencial para asistir en el uso costoefectivo de los recursos dirigidos a los problemas prioritarios de salud. La efectividad es la medida del grado con el cual los objetivos deseados son cumplidos haciendo un uso eficiente de los recursos. En materia de salud se refiere a como ha mejorado o empeorado el estado de salud de una población en relación a una intervención y por consecuente al uso de recursos. De esta manera el concepto se refiere al vínculo que existe entre la producción del efecto y el desempeño del mismo.

Si bien es cierto existen estudios sobre el costoefectividad para IRCT en paises de ingreso alto ${ }^{3,10,20}$, donde los resultados orientan hacia la promoción del trasplante renal, sin entrar a mayor detalle, tambien es cierto que en paises de ingreso medio como México, este tipo de estudios escazamente han sido desarrollados, y por lo tanto los programas de atención se organizan de acuerdo a los resultados de otros paises. En este caso, se trata de retomar metodologías de análisis desarrolladas en paises de ingreso alto y aplicarlas a las condiciones de salud y de los sistemas de salud de países de ingreso medio como México.

Para comparar tratamientos alternativos se pueden considerar numerosas medidas de éxito como indicadores inmediatos (tasa de recurrencia, tasa de mortalidad, sobrevida), el problema radica en que los resultados a que hacen referencia los indicadores pueden no ser homogéneos. La selección de los criterios de efectividad responde al tipo de estudio y es preferible que estén validados en el mismo tipo de padecimientos. En este estudio, tomando en cuenta su aplicación y validación en México, se han seleccionado como criterios de efectividad la probabilidad de sobrevida de los pacientes y los años de vida ajustados por calidad (QALY, Quality Adjusted Life Years) para cada alternativa de intervención ${ }^{17}$.

En lo relativo a la evaluación de los costos de cada intervención, por dificultades conceptuales y metodológicas, existen pocos estudios que incluyan información sobre los costos de producir servicios, sobre todo por el hecho de que no existe una clasificación idónea que permita analizar los costos para ambos actores del mercado de servicios médicos: el proveedor y el consumidor ${ }^{1}$.

En efecto, trátese del consumidor o del proveedor, el concepto económico de costos surge de la noción 
de usos alternativos de los recursos financieros ${ }^{9}$. El costo económico de una unidad de recurso es el beneficio que se obtendría en un mejor uso alternativo. Este concepto debe ser contrastado con el concepto estrictamente contable de costo que está relacionado con las salidas de caja por unidad de recurso (gasto). Por otra parte, las estimaciones contable y económica de costos de salud pueden coincidir, pero esto no es lo más frecuente, pues no se incluye en la perspectiva contable el concepto de costo de oportunidad y generalmente sólo se incluye lo referente a costo recurrente.

Para el caso de los servicios de salud es importante considerar que los costos se definen como el monto de recursos económicos que se invierten durante cualquier evento de atención a la salud ${ }^{5,19}$. Tomando en cuenta esta problemática en materia de evaluación económica, para este estudio, los costos se determinaron a partir de la identificación del costoeconómico de cada intervención en un hospital de referencia nacional perteneciente al sector público y dirigido a población no asegurada. Finalmente, a partir de los costos y la efectividad de cada intervención se procedió matemáticamente a determinar el coeficiente de costo-efectividad, identificando así la intervención más costo-efectiva y sus implicaciones en materia de eficiencia y efectividad en el uso de los recursos financieros.

\section{MÉTO DO}

Se trató de un estudio de caso mediante un diseño transversal de tipo evaluativo. El universo de estudio incluyó a todos los pacientes que reciben servicios para IRCT del hospital de referencia, donde se ubica una unidad de hemodiálisis, existe un programa de DPCA y se realizan trasplantes renales. La población de estudio estuvo constituída por 40 pacientes del mismo hospital, mismos que fueron seleccionados de acuerdo a los siguientes criterios de inclusión:

- Estar actualmente en tratamiento para IRCT en el hospital de estudio con alguna de las intervenciones seleccionadas.

- Haber sido trasplantados o que hayan estado con alguna intervención de diálisis por lo menos un año y máximo 6 años .

- Que la causa de la IRCT haya sido primaria, es decir que no se pueden incluir pacientes con patologías agregadas como diabetes o lúpicos.

- La edad de los encuestados debió estar entre los 20 y 45 años de edad (adultos jóvenes).

- Aprobación del paciente y conformidad con la entrevista directa.

Se utilizaron fuentes bibliográficas para la sobrevida de los pacientes en DPCA y trasplantados. A este respecto se consiguieron datos actualizados, desafortunadamente no existe información concernientes a la hemodiálisis debido a que la unidad de hemodiálisis sólo atiende a pacientes próximos a trasplantarse o pacientes que estén en DPCA que por alguna complicación se hemodializan temporalmente.

Para el caso del trasplante se utilizó la sobrevida del injerto y no la del paciente para no mezclar sobrevidas atribuibles a otros procedimientos a los que pudiera haber estado sometido el paciente además del trasplante. Para actualizar aún más la información referente a la sobrevida del injerto, se calculó la sobrevida por el método actuarial utilizando la información de la base de datos del departamento de trasplantes del hospital mencionado de los 10 años anteriores a julio de 1995 (fecha en la que se actualizó la información). Se incluyó a todos los pacientes trasplantados sin importar si el donador era vivo o cadavérico, número de haplotipos compartidos o diagnóstico inicial.

Con la disponibilidad de datos y utilizando el mismo método, se calculó la probabilidad de sobrevida de los injertos trasplantados desde 1967 (cuando se inició el programa de trasplantes) hasta julio de 1985 para poder hacer una comparación con la probabilidad de sobrevida de los injertos trasplantados de 1985 a 1995 con el fin de mostrar los avances alcanzados en materia de los años de vida ganados.

El Índice de Rosser se utilizó para el cálculo de los QALYs para cada procedimiento. El índice identificó dos dimensiones incapacidad y tensión; estas dos dimensiones se operacionalizaron en un cuestionario que se aplicó bajo

Tabla 1- Niveles del Índice de Rosser*.

\begin{tabular}{cc}
\hline \multicolumn{1}{c}{ Incapacidad } & \multicolumn{1}{c}{ Tensión } \\
\hline I. Sin incapacidad & A. Sin tensión \\
II. Ligera incapacidad social & B. Ligeramente \\
III. Severa incapacidad social & tensionante \\
y/o ligera incapacidad & C. Moderadamente \\
laboral. Capacidad de & tensionante \\
hacer trabajos caseros & D. Severamente \\
IV. Capacidad severamente & tensiosante \\
limitada de desempeño & \\
laboral. Capacidad de & \\
hacer trabajos ligeros & \\
caseros y salir de compras & \\
V. Incapaz de mantener & \\
un empleo. Incapaz de & \\
seguir un plan de estudios. & \\
Seniles delimitados a su & \\
hogar sin poder salir & \\
hacer compras. Amas de & \\
casa capaces sólo de & \\
desempeñar algunas & \\
tareas caseras. & \\
VI. Delimitación a una silla \\
de ruedas. Capacidad de \\
moverse alrededor de la \\
casa sólo con la ayuda \\
de un asistente
\end{tabular}

* Clasificación de Rosser en Gudex Cn, 1986. 
la técnica de entrevista directa con los pacientes seleccionados. Los indicadores incluídos en dicho cuestionario representaron 8 niveles de incapacidad y 4 de tensión que se utilizaron para describir 29 estados de enfermedad o salud (Tabla 1). El Índice puede tener valores entre 0 y 1 ; el 1 representa un estado de salud óptima (completa movilidad y ninguna tensión), mientras que el cero representa la muerte (Tabla 2). El cuestionario, fue validado para el contexto mexicano, y se aplicó a los pacientes bajo los tres diferentes procedimientos. Las preguntas definitivas guardan estrecha similitud, en la medida de lo posible, a la descripción de los 29 estados de salud del Índice de Rosser ${ }^{13}$.

El cuestionario incluyó una primera batería de preguntas acerca de la movilidad, que incluye desde la posibilidad de desplazarse en ambientes de interiores y exteriores sin ayuda de nadie hasta requerir pasar parte del día en cama. La siguiente batería de preguntas se relaciona al cuidado personal del paciente (bañarse, vestirse, comer, etc.). También se incluyeron reactivos sobre actividades cotidianas, sociales y forma de funcionar en sociedad con el estado de enfermedad de cada paciente. Además, existió un apartado para sentimientos generados por el estado de enfermedad del paciente (tristeza, frustración o enojo). La última batería de preguntas, solicitó al paciente calificar el grado de estres que le provoca su estado de enfermedad y los aspectos que más le molestan del mismo.

No existió un período de seguimiento estricto, la valoración de cada indicador de efectividad se hizo através de un corte transversal en el tiempo mediante entrevistas directas con los pacientes y através del método actuarial, con la información de archivos clínicos y tomando en cuenta desde el paciente mas antiguo hasta el mas reciente. El período de referencia para las entrevistas fué la primera y segunda semana de diciembre de 1995.

Para la determinación del costo de cada intervención se identificaron los insumos, los costos de los insumos, las funciones de producción por manejo de caso promedio y finalmente el costo por manejo de caso. Para identificar el tipo y cantidad de insumos por función de producción, se partió del protocolo que el hospital de estudio utiliza para el manejo de los pacientes. La información generada se validó con profesionales del área y para la cuantificación

Tabla 2- Índice de Rosser*.

\begin{tabular}{ccccc}
\hline \multicolumn{5}{c}{ Valores de Rosser transformados de los 29 estados de I/T } \\
\hline Incapacidad & Tensión \\
I & 1,000 & 0,995 & 0,990 & 0,967 \\
II & 0,990 & 0,986 & 0,973 & 0,932 \\
III & 0,980 & 0,972 & 0,956 & 0,912 \\
IV & 0,964 & 0,956 & 0,942 & 0,870 \\
V & 0,946 & 0,935 & 0,900 & 0,700 \\
VI & 0,875 & 0,845 & 0,680 & 0,000 \\
VII & 0,677 & 0,564 & 0,000 & $-1,486$ \\
VIII & $-1,028$ & - & - & - \\
\hline *Matriz de valores de Rosser ${ }^{11}$ & & &
\end{tabular}

* Matriz de valores de Rosser ${ }^{11}$ de insumos en el proceso terapeútico y de intervención quirúrgica, la información se complementó con la literatura ${ }^{4,18,21,23}$. Se realizaron entrevistas con expertos clínicos para identificar insumos y funciones de producción faltantes en el protocolo, particularmente en cuanto al seguimiento de la intervención. Los detalles sobre esta evaluación económica aparecen en Arredondo et al. ${ }^{2}, 1998$.

En todos los casos la guía de base la constituyeron los protocolos de estudios y procedimientos en el manejo de intervenciones para la IRCT, así como la historia natural de la enfermedad de acuerdo a cada intervención. Los costos al proveedor se obtuvieron de los distintos centros de costos existentes y los costos al consumidor, se obtuvieron de los tabuladores de cuotas de recuperación de costos. El período de referencia monetaria fué la última quincena de noviembre de 1995, con tipo cambiario de $\$ 7.15$ pesos mexicanos por $\$ 1.0$ dóllar estadounidense. El coeficiente costo-efectividad se calculó a través de la relación de indicadores de efectividad vs. indicadores de costo de manejo anual de caso de cada intervención.

\section{RESU LTAD O S}

\section{Criterios de Efectividad}

\section{Probabilidad de la Sobrevida}

Los cambios en la probabilidad de la sobrevida entre modalidades de tratamientos fueron obtenidos directamente de los pacientes estudiados y de otros estudios referidos, así cabe destacar, que el trasplante renal si bien no es sustancial para el primer año, si lo es para el tercer y sexto año (Tabla 3). Mientras que la probabilidad de la sobrevida disminuye pro-gresivamente con el tiempo en la DPCA, el TR observa un notorio aumento entre el primer y tercer año.

Por otro lado, el seguimiento a lo largo del tiempo del TR en el hospital de estudio, demuestra claramente una mejora sustancial en la probabilidad de la sobrevida de injertos trasplantados. La Tabla 4 se basa en los resultados de una base de datos histórica del departamento de trasplantes del hospital ya referido y en los pacientes de este estudio para reportar las probabilidades de sobrevida en tres períodos de tiempo: 1967-1985, 1985-1995. Esta base de datos consiste de 182 y 234 trasplantes para

Tabla 3 - Probabilidad de sobrevida para diálisis peritoneal contínua ambulatoria (DPCA) y trasplante renal

\begin{tabular}{ccc}
\hline Años & DPCA* & Sobrevida del injerto \\
\hline 1 & $86,2 \%$ & $89,9 \%$ \\
3 & $66,9 \%$ & $79,6 \%$ \\
6 & $37,5 \%$ & $75,8 \%$ \\
\hline
\end{tabular}


el primer y segundo período respectivamente, sumando un total de 416 trasplantados de 1967 a 1995 (Figura).

Así, observamos que el primer período se distingue por tener la más baja probabilidad de sobrevida llegando a $38.35 \%$ a los diez años. También resalta este período por reportar la mayor diferencia entre el primer y tercer año, es decir de 13.48 puntos porcentuales. En el período de 1985-1995 se encuentran sensibles aumentos de la probabilidad de sobrevida entre los diferentes años, lo que hace evidente las mejorías del programa de trasplantes de dicho hospital.

\section{Calidad de Vida}

Los cambios en la calidad de vida de pacientes con IRCT con respecto a las alternativas de tratamiento son estadísticamente significativos. ElTR ofrece la mayor calidad de vida en cuanto a incapacidad y tensión (QALY $=0,978, \alpha=0,002$ ). Esto es, el TR reporta mejor calidad de vida en comparación con los otros tratamiento en relación a: (1) mejor movilidad general, ya sea en dentro o fuera de la casa-habitación del paciente o sin necesitar la ayuda de alguien para desplazarse de un lugar a otro; (2) el estado de salud del paciente afecta en menor grado su vida social, pasatiempos y vida sexual; (3) su estado de salud los llevó a experimentar en menor grado sentimientos como: tristeza, depresión, ansiedad, preocupación, dolor, angustia, enojo, resentimiento, soledad, etc. y (4) los pacientes reportan que su estado de salud los mantiene menos tensionados que antes del tratamiento.

Los QALY entre la DPCA y la hemodiálisis no reportan significancia estadística. El número de pacientes es muy reducido para la DPCA y la HD, $n=15$ y $n=4$, respectivamente. Sin embargo, cuando se toman ambos tratamientos y se agrupan como diálisis, entonces si se observa una significancia estadística entre la diálisis y el TR (diálisis $=0,88, \alpha=0,002$ ). Ejemplificando el comportamiento de cada intervención en 5 años por medio de proyecciones, con una tasa de descuento de $5 \%$, para un período de cinco años, el paciente sometido a TR tendrá una QALY de 4,44, mientras que el paciente en DPCA obtendrá un QALY de 3,99.

\section{Manejo Anual de Caso y Costos}

-Manejo anual de caso para diálisis peritoneal ambulatoria (Insumos y/o funciones de producción).

La intervención incluyó un curso de capacitación para la aplicación de diálisis en el domicilio del paciente, material y equipo fijo anual, material y equipo para cambio de línea dos veces

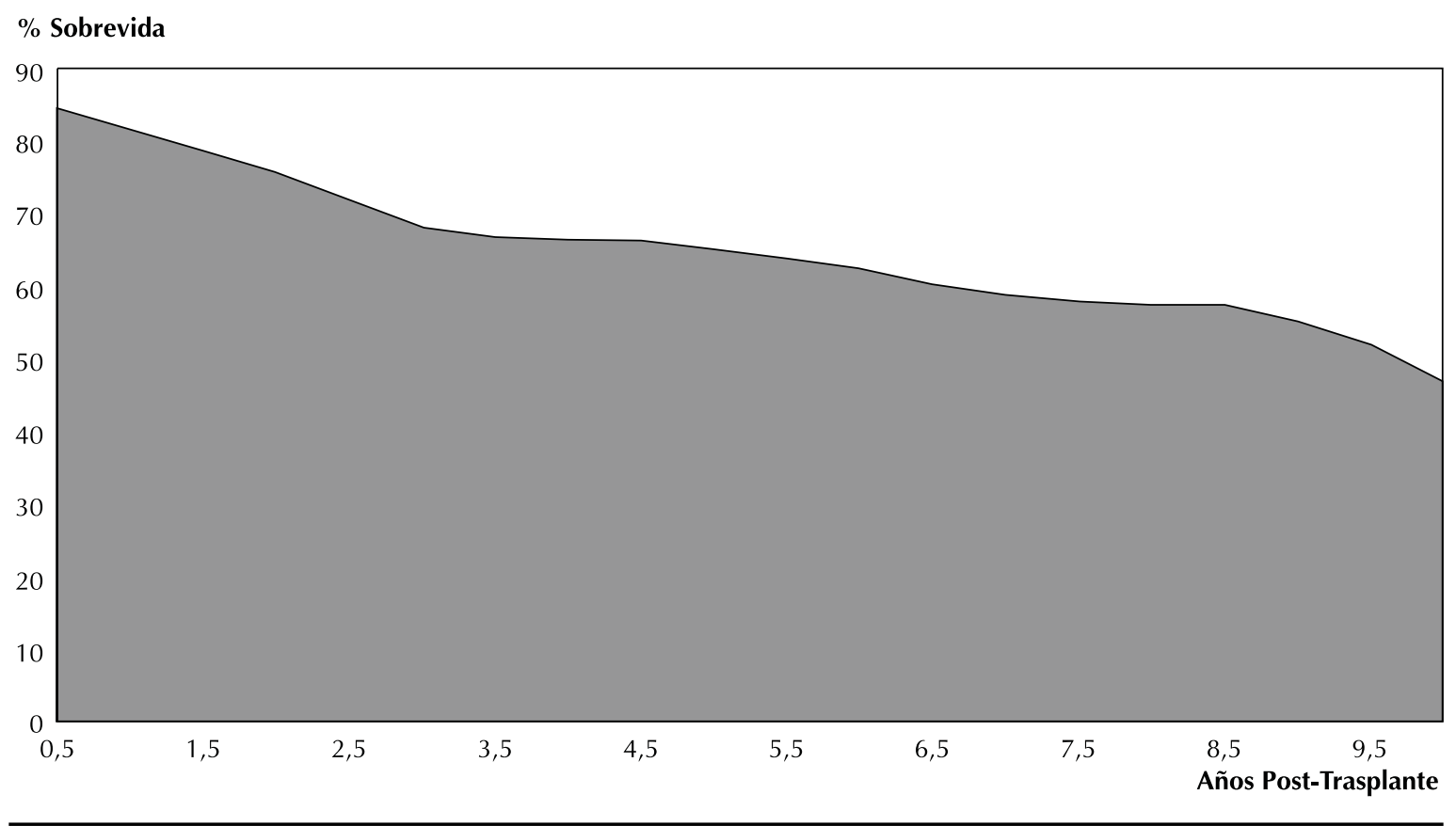

Fuente: Cálculos hechos por el autor con información de la base de datos del depto. de trasplante del IN N SZ. Figura - Probabilidad de sobrevida del injerto de trasplante renal. Datos de 1967-1995. 
por año, 1.460 eventos de diálisis por año, estudios de laboratorio trimestrales, cuatro consultas de control y seguimiento.

-Manejo anual de caso para hemodiálisis (Insumos y/o funciones de producción).

La intervención incluyó 156 hemodiálisis por año, una cirugía para fístula arteriovenosa cada dos años, estudios de laboratorio mensuales, estudios trimestrales y cuatro consultas de control y seguimiento.

-Manejo anual de caso para trasplante (Insumos y/o funciones de producción).

La intervención incluyó para donador: ocho consultas de especialidad, hospitalización, cirugía, estudios clínicos, de laboratorio y gabinete. Para receptor: cirugía renal, quince interconsultas de especialidad, estudios de histocompatibilidad, clínicos, de laboratorio y gabinete, hospitalización preoperatorio, cirugía de trasplante, estudios post trasplante, estudios de control y seguimiento, medicamentos temporales post trasplante y medicamentos permanentes.

- Costos de manejo anual de caso de cada intervención.

Para las tres intervenciones de estudio, cada uno de los componentes del costo se trabajaron a detalle identificando insumos, funciones de producción, costos de los insumos y costos de los servicios al consumidor de acuerdo al tabulador de la institución de estudio. Los costos por evento resultantes en US dóllares fueron: DPCA (\$3.71), Hemodiálisis (\$57.95) y TR $(\$ 8,778.32)$. Estos costos por evento constituyeron la base de información para identificar los costos de manejo anual por intervención.

En relación al manejo anual de diálisis peritoneal, fué necesario agregar los costos del material y equipo fijo para los eventos de diálisis peritoneal, así como el material y equipo para cambio de línea dos veces al año. Se incluyeron 1.460 eventos de diálisis por año, así como los estudios de laboratorio, estudios de gabinete e interconsultas de control y seguimiento durante un año; resultando un costo total por año de $\$ 5,643.07$ por cada paciente en el programa de diálisis peritoneal ambulatoria.

En cuanto al costo de manejo anual para pacientes con hemodiálisis, fue necesario agregar el costo de una cirugía para fístula arteriovenosa, 156 eventos de hemodiálisis por año, estudios de laboratorio, estudios de gabinete y consultas de control y seguimiento por año. El resultado del costo de manejo anual en pacientes con hemodiálisis fue $\$ 9,631.60$.

El costo de manejo anual de caso para trasplante se desarrolló en dos etapas, en la primera etapa se costeó el costo del trasplante per se y en la segunda etapa se determinó el costo de manejo anual de caso, considerando el trasplante como una inversión con un promedio de sobrevida de seis años y aplicando una tasa de interés. Para la primera etapa ( costo del trasplante) se incluyeron estudios pretrasplante al donador y receptor, manejo preoperatorio del receptor, cirugía del donador, cirugía del receptor, estudios post-trasplante, medicamentos post-trasplante, medicamentos permanentes, así como estudios de laboratorio, estudios de gabinete, medicamentos y consultas de control y seguimiento del trasplante durante el primer año. El resultado de esta primera etapa de costos del trasplante fue de $\$ 11,199.03$.

Para la segunda etapa del costo de trasplante (costo de manejo anual) fue necesario determinar el costo anual de interconsultas de control y seguimiento, así como estudios de laboratorio y gabinete y medicamentos permanentes para los siguientes 5 años. El resultado del costo anual se calcula en base a que una vez hecho el trasplante que resultó en \$ 584.54, a este costo se le restó al costo del primer año, pues es un costo que ya se realizó, de tal forma se realizo la siguente operación: $(11,199.03)-(584.54)=$ $\$ 10,614.49$, y el resultado se tomó como equivalente al monto de inversión que se está realizando a 6 años con una tasa de interés al $10 \%$. Por lo tanto el costo de manejo anual de caso con trasplante (CMT), finalmente se obtuvo de la siguiente manera:

CMT = costo anual + costo de inversión $[(\mathrm{i}(1+\mathrm{i}) \mathrm{n}) /$ $((1+\mathrm{i}) \mathrm{n}-1))]$

donde $\mathbf{i}$ es la tasa de interés y $\mathbf{n}$ es el número de años que durará la inversión, el control y el seguimiento. Entonces el costo de manejo anual con trasplante resulta en:

$\mathrm{CMT}=[(584.55)+10,614.49[(.1771561) /$ $(.771561)]=\$ 3,021.63$

que es el costo real de manejo anual de caso con trasplante renal.

Es necesario resaltar que los costos de cada una de las intervenciones variaron significativamente al determinar el costo de manejo de caso anual. En efecto, el costo de diálisis peritoneal ambulatoria por evento de \$3.71 se incrementó a \$ 5,643.07 al momento de identificar el costo de manejo anual para diálisis peritoneal ambulatoria; el costo de hemodiálisis por evento de $\$ 57.95$ igualmente se incrementó a \$9,631.60 al momento de identificar el costo de manejo anual de casos de pacientes con hemodiálisis. Por otra parte el costo de trasplante per se, de \$ 8,778.32, disminuyó a \$ 3,021.63 al calcular el costo de manejo anual de pacientes con trasplante. 


\section{Coeficiente Costo-efectividad}

Utilizando los resultados de costo de manejo anual de caso, así como los resultados sobre la efectividad de cada intervención, se calcularon los coeficientes costo-efectividad, cuyos resultados aparecen en la Tabla 5 .

De acuerdo a estos resultados, la intervención mas costo-efectiva es la que resultó con el menor coeficiente, siendo en esta caso el TR, seguido de la CAPD y la hemodiálisis con el coeficiente mayor y por lo tanto como la intervención menos costo-efectiva.

\section{DISCUSIÓN}

Antes de entrar al detalle de los resultados, es necesario hacer algunas acotaciones en términos de la validéz y representatividad de los hallazgos sobre efectividad y costos de cada intervención. En primer lugar, hay que resaltar que se trató de un estudio de caso y que por lo tanto el tamaño de la muestra para estimar la calidad de vida en QALYs, especialmente para la hemodiálisis no permite hacer inferencias que representen a la población de pacientes a nivel nacional con IRCT, pero sí para la población que demanda el tipo de intervenciones de estudio en el hospital de análisis de este estudio.

$\mathrm{Ni}$ en términos de los resultados de efectividad ni en términos de los resultados sobre costos se puede hablar de representatividad en este tipo de estudios, aún cuando se hubiera trabajado con una muestra representativa a nivel nacional. Particularmente, los

Tabla 4 - Probabilidad de sobrevida del trasplante renal en periodos de tiempo*.

\begin{tabular}{cccc}
\hline $\begin{array}{c}\text { Erreur! } \\
\begin{array}{c}\text { Signet non } \\
\text { défini. } \\
\text { Años }\end{array}\end{array}$ & $\begin{array}{c}\text { De 1967 } \\
\text { a 1985 }\end{array}$ & $\begin{array}{c}\text { De 1985 } \\
\text { a 1995 }\end{array}$ & $\begin{array}{c}\text { Total } \\
1967 \\
\text { a 1995 }\end{array}$ \\
\hline 1 & 71,23 & 89,90 & 81,52 \\
3 & 57,75 & 79,56 & 69,56 \\
6 & 51,28 & 75,80 & 64,03 \\
10 & 38,35 & 58,77 & 47,21 \\
\hline
\end{tabular}

* Cálculos apoyados en la base de datos del Departamento de Trasplantes del Hospital de la Secretaria de Salud, 1995. costos, en términos absolutos, de producir servicios de salud en cualquier evaluación económica de servicios de atención médica, sólo se pueden referir al hospital objeto de evaluación ya que dichos costos varían de un hospital a otro, aún dentro del mismo país o institución.

Los criterios de inclusión deliberadamente se seleccionaron para excluir pacientes con un estado de salud y psicológico incompatible con el grado de cooperación necesaria para desarrollar el estudio, por lo que el estudio está limitado en cuanto a la interpretación de los resultados en la población general de urémicos. Sin embargo, la relativa homogeneidad del grupo de pacientes da la confianza de que las diferencias estadísticas observadas entre los grupos realmente demuestran el efecto de cada alternativa de tratamiento.

Los resultados obtenidos en cuanto a los costos por intervención sólo se aplican para el consumo de servicios de salud en centros de atención de tercer nivel. No obstante, las frecuencias relativas del costo de cada insumo o función de producción, en relación al costo total por evento o manejo anual, seguramente son similares en centros de atención de otras instituciones públicas para pacientes con insuficiencia renal crónica.

Por otra parte, hay que resaltar que el costo de cada intervención, se refiere al costo promedio de manejo de caso sin tomar en cuenta las complicaciones que puedan presentarse en la práctica de cada centro hospitalario, incluso de cada paciente, pues hay que recordar que hablando de saludenfermedad, existen enfermos y no enfermedades. Tomando en cuenta lo anterior, la aproximación de la valoración de costos realizada, puede utilizarse como el costo mínimo por intervención, mientras que el costo máximo dependerá de las complicaciones que se requieran costear.

En relación a la efectividad, los resultados del estudio sugieren que entre las alternativas de tratamiento para la IRCT, el TR reporta la mayor efectividad en cuanto a criterios de sobrevida y calidad de vida. La mayor probabilidad de vida del paciente trasplantado con respecto al paciente en DPCA se debe a que el trasplante es un tratamiento curativo, mientras

Tabla 5 - Coeficientes de costo-efectividad para cada intervención.

\begin{tabular}{lccc}
\hline Intervención & Costo en US\$ & Índice de Rosser* & Coeficiente C-E \\
\hline DPCA & $5,643.07$ & 0,8794 & $6.416,95$ \\
Hemodiálisis & $9,631.60$ & 0,8640 & $11.147,68$ \\
Trasplante renal & $3,021.63$ & 0,9783 & $3.088,69$ \\
\hline
\end{tabular}


que las otras dos opciones se mantienen como tratamientos crónicos.

Los resultados de costo-efectividad de este estudio, además de coincidir con los resultados de otros países ${ }^{8,12,24}$, constituyen una base de información más válida y detallada, no sólo para el análisis de costo-efectividad, sino también para diferentes análisis de costo utilidad que se requieran hacer en relación al costo y financiamiento de la producción de servicios para IRCT, o bien en relación a posibles ajustes al protocolo de la atención médica para cada una de las intervenciones de estudio.

No se reportan resultados de la probabilidad de sobrevida de pacientes en hemodiálisis, pero por resultados de otras publicaciones es de esperarse, como lo mostraron los hallazgos de este estudio, que también la probabilidad de sobrevida del injerto del trasplante sea mayor que la probabilidad de vida del paciente en hemodiálisis. Por otra parte, las pruebas estadísticas no paramétricas reportaron diferencias significativas en la calidad de vida entre las muestras de pacientes en diálisis y trasplantados, situación que no sucede entre las muestras de pacientes en DPCA y hemodiálisis.

Las mejoras en calidad de vida del paciente trasplantado con respecto al dializado se deben principalmente a la mayor movilidad y menor tensión del paciente. Los pacientes trasplantados tienen mayores posibilidades de realizar un trabajo remunerado puesto que no interrumpen sus actividades cotidianas para dializarse. Por otro lado, los pacientes trasplantados sufren de menos tensión ya que reportan estar menos deprimidos, preocupados, ansiosos y/o insatisfechos que los pacientes en diálisis.

En relación a los costos resultantes por evento, puede decirse en primera instancia, que como eventos aislados, la diálisis peritoneal ambulatoria y la hemodiálisis resultaron en un costo insignificante en relación al costo del trasplante. Sin embargo al identificar el costo de manejo anual para cada intervención los resultados cambiaron de manera significativa, ubicando el costo de manejo anual de trasplante como el menos costoso, seguido del costo de manejo anual con diálisis peritoneal como más costoso y más aún el de la hemodiálisis.

El estudio realizado es el caso típico donde la evaluación económica debe considerar la perspectiva clínica y de mercado para identificar los servicios requeridos para una intervención específica en un período determinado. De otra manera se corre el riesgo de tomar decisiones a partir de los resultados de costo por evento sin tomar en cuenta dos aspectos relevantes: la demanda real del servicio y las repercusiones económicas del manejo anual por intervención. En todo caso las decisiones al margen de estos dos aspectos, incrementarán la ineficiencia en los patrones de asignación de recursos y por consecuente, la ineficiencia en el uso de recursos para producir servicios y en el financiamiento de los mismos.

En términos de eficiencia económica, sin tomar en cuenta los criterios de efectividad, el trasplante renal resulta en un menor costo, seguido de la diálisis peritoneal ambulatoria y de la hemodiálisis. Desde una perspectiva económica y de efectividad clínica y sobre todo a partir de los resultados del coeficiente costo-efectividad, puede decirse de manera más contundente que el trasplante renal, efectivamente debería ser la intervención de mayor promoción y aplicación en cuanto a las tres intervenciones de estudio.

Los resultados aquí obtenidos pueden proporcionar argumentos para ampliar el programa de trasplantes en hospitales de tercer nivel de atención y a su vez sugerir la ampliación de programas de procuración de órganos cadavéricos. Por otra parte, debe tomarse en cuenta que los resultados obtenidos se refieren a los pacientes que demandaron servicios en un hospital cuyos niveles de eficiencia y calidad son de los más aceptados en el contexto del sector salud de México. Por lo tanto, los resultados de costoefectividad, pueden diferir substancialmente dependiendo de los niveles de eficiencia, efectividad y calidad con que operen otros centros de atención.

Finalmente, dentro del actual contexto de reformas del sector y tomando en cuenta dos de los principios rectores de la producción de servicios de atención médica: las necesidades de salud que presenten los cambios en el perfil epidemiológico y la escasez de recursos para satisfacer la demanda que generan dichas necesidades, los resultados del coeficiente costo-efectividad identificado desde una perspectiva clínica y económica, constituyen un aporte relevante para la búsqueda y el logro de la eficiencia de los recursos que se asignan para producir servicios de salud para pacientes con IRCT, cuyas demandas están en competencia con otras enfermedades crónicas e infecto-contagiosas. 


\section{REFERENCIAS}

1. ARREDONDO, A. \& DE ICAZA ESTEBAN, D. T. Costos de servicios de salud en diferentes instituciones del sector público y privado en México. Salud Pública Méx., 36: 437-45, 1995.

2. ARREDONDO, A. et al. Costos de intervenciones para pacientes con insuficiencia renal crónica. Rev. Saúde Pública, 32:255-61, 1998

3. BADIA, X. et al. Reliability of the Spanish version $f$ of the Nottingham Health Profile in patients with stable end-stage renal disease. Soc. Sci. Med., 38: 153-8, 1994.

4. BORDES, A. et. al. Twenty four year experience in kidney transplantation at one single institution in México City.

Transpl. Proc., 24: 1992.

5. BRENZEL, L. Application of cost-effectivenss analysis to decision-making in the health sector of developing countries. Washington, World Bank, technical report, 1993. p. 79-82.

6. CHAVEZ, R. et al. Diálisis peritoneal continua ambulatoria: experiencia de los últimos 6 años en el INNZS. México, 1995. [Apresentada a $44^{a}$ Reunión Anual del Instituto Mexicano de Investigaciones Nefrológicas, Ixtapa Zihuatanejo, 1995].

7. DE ICAZA, E. et al. Changes in the quality of life of patients with end-stage renal disease treated with highefficiency bicarbonate hemodialysis in Mexico. Renal Failure, 19: 99-110, 1997.

8. DE VECHI, A. \& SCALAMOGNA, A. Well being in patient on CAPD and hemodialysis. Int. J. Artif. Organs, 17:473-7, 1994.

9. DRUMMOND, M. F. Principios de evaluación económica en asistencia sanitaria. Madrid, Instituto de Estudios Laborales y de la Seguridad Social, 1983.

10. EVANS, R. et al. The quality of life of patients with endstage renal disease. N. England J. Med., 312: 553-9, 1985.

11. FRENK, J. et al. Economía y salud: propuestas para el avance del sistema de salud en México: visión de conjunto. $2^{\mathrm{a} e d . ~ M e ́ x i c o ~ F u n d a c i o ́ n ~ M e x i c a n a ~ p a r a ~ l a ~ S a l u d, ~}$ 2: 1996. v.2. p.14-31.
12. GOKAL, R. Quality of life in patients undergoing renal replacement therapy. Kidney Int., 43 (Suppl. 1996.v.2, p. 14-3. 40): s23-s27, 1993.

13. GUDEX, C. QALYS and their use by the health service. Center for Health Economics, University of York, 1986. (Discussion paper, 20).

14. HERNÁNDEZ, G. et. al, Algunas reflexiones sobre la insuficiencia renal crónica terminal en México. Gac. Med. Méx., 131: 459-63, 1995.

15. JAMISON, D. \& MOSLEY, H. Disease control priorities in developing countries: health policy responses to epidemiological change. Am. J. Public Health, 81:15-22, 1991.

16. JAMISON, D. et al. Disease control priorities for developing countries. Washington D.C. Oxford University Press/World Bank, 1993.

17. LOWRIE, E. \& HAMPERS, L. The success of medicare s end stage renal disease program. $N$. England J. Med., 305: 434-8, 1981.

18. MILLS, A. The financing and economics of hospitals in developing countries: key issues and research questions. London, World Bank-Population and Human Resources Department, 1990. (PHN Technical Notes).

19. NISSENSON, A. Measuring, managing and improving quality in the end-stage renal disease treatment setting: peritoneal dialysis. Am. J. Kidney Dis., 24:368-73, 1994.

20. NOLPH, K. et al. Current concepts: continuous ambulatory peritoneal dialysis. N. Engl. J. Med., 318:1595-600, 1988.

21. RAMOS, L. et al. Estudios egresos hospitalarios de diabetes mellitus durante 7 años a nivel internacional en el Instituto Nacional del Seguro Social. Rev. Méd. IMSS, 27: 141-6, 1989.

22. SCIENTIFICAMERICAN OF MEDICINE. Nefrología. México, Editora Científica Medica Latinoamericana, 1994. v. 2.

23. SINGH, S. et al. Multicenter study of change in dialysis therapy-maintenance hemodialysis to continuous ambulatory peritoneal dialysis. Am. J. Kidney Dis., 19: 246-51, 1992. 